First publ. in: Two-Dimensional Electron Systems / ed. by Eva Y. Andrei. Kluwer, 1997, pp. 317-339

\title{
NONLINEAR EFFECTS - THE MULTIELECTRON DIMPLE
}

\author{
P. LEIDERER \\ University of Konstanz \\ D-78434 Konstanz, Germany
}

\section{Introduction}

In the field of 2-dimensional electron systems the surface of liquid helium has proven as a nearly ideal substrate for surface state electrons (SSE) ${ }^{1}$. In the previous chapters this surface could be considered to a good approximation as being completely inert and smooth, except for surface excitations (ripplons), which act as the dominating source for SSE scattering at low temperature, where scattering of the electrons by gas atoms diminishes. An influence of the electrons on the helium substrate, however, could so far very well be neglected. By contrast, we discuss in this chapter phenomena at high charge densities and high electric holding fields, where the electrons, due to the coupling of the SSE system to (long wavelength) surface excitations, have a significant influence on the substrate. This gives rise to an interesting variety of phenomena at the helium surface, which then in turn also affect the electron system itself.

Already in the very early investigations of SSE on helium by Williams and Crandall $^{2}$ it was observed that the liquid surface is slightly depressed in the region under the electron sheet due to the electrostatic pressure of the surface charges. As will be shown below, charging the surface does not only lead to this static effect, but also causes changes in the dynamics of the system. Since the electric forces counteract the restoring forces for a flat surface - surface tension and gravity - the frequency of surface waves will be reduced, an effect which is most pronounced at a particular wave vector $q_{c}$, given by the inverse capillary length. As the charge density grows, this "ripplon softening" is enhanced, until eventually the surface becomes unstable above a maximum supportable electron density $n_{c}$ of about $2 \times 10^{9} \mathrm{~cm}^{-2}$. Deep troughs then form in the surface, whence the electrons punch through in the form of multielectron bubbles and move towards the positively charged electrode immersed in the helium.

A completely different scenario is observed when the surface is not charged to the maximum density, but to only ten percent of $n_{c}$ or less. In this case the instability, which then occurs at a somewhat higher external electric field, does not yield chargeinduced deformations of the surface which grow continuously, but rather these deformations become stabilized at a certain amplitude as a result of nonlinear effects. The surface then displays a periodic pattern, where the electrons are localized in a regular array of pockets, or "dimples", which constitute a macroscopic analogue of the 
microscopic Wigner crystal discussed in chapter 4. These many-electron dimples, each of which contains up to $10^{7}$ electrons in a spot with a typical diameter of the order of $1 \mathrm{~mm}$, also exist as individual entities at the helium surface and can also be considered as a macroscopic version of the one-electron polaron (see chapter 12 of this volume).

The various stages of the electrohydrodynamic (EHD) instability caused by electrons at helium surfaces are also interesting from the point of view of phase transitions and critical phenomena, because they display in a transparent way the fundamental mechanisms, as they are also relevant, e.g., for structural phase transitions or spinodal decomposition. Beyond this model aspect, however, an understanding of the instability is also essential if one tries to reach high electron densities above $n_{c}$, where quantum effects due to the Fermi nature of the electrons should eventually come into play. One of the most striking examples is the predicted melting of the Wigner crystal towards the degenerate 2D Fermi gas around $10^{12} \mathrm{e} / \mathrm{cm}^{2}$. Knowing the origin of the instability one can employ additional forces in order to stabilize the surface, like capillarity (by using helium surfaces pinned in a confined geometry, for instance supplied by solid structures in the $\mu \mathrm{m}$ range ${ }^{3}$ ) or van der Waals forces (by using thin helium films as a substrate for the SSE system) and thus reach electron densities in the quantum range of the SSE phase diagram.

In the following, we treat in section 2 the equation of motion of a charged interface and the softening of the interfacial waves. Section 3 is devoted to the development of the periodic surface deformation and the properties of the dimple lattice. In section 4, individual multielectron dimples are investigated with respect to their stability, shape, and charge distribution. Section 5 deals with the development of the surface instability at electric fields well above the critical field, and section 6 with the multielectron bubbles forming in the liquid helium upon the collective punchthrough process of the charges at the surface. Finally, in section 7 the stability of SSE on helium films is considered.

\section{Excitation Spectrum of a Charged Surface: The Soft Ripplon Mode}

Neutral surface. Before discussing the influence of charges on the spectrum of surface excitations at the liquid helium surface, we briefly outline the situation for an uncharged surface. The motion of the surface, at rest located in the $x-y$ plane, is governed by ${ }^{4}$

$$
\left(\rho_{*}+\rho_{+}\right) \partial \phi / \partial t=-\left(\rho_{-}-\rho_{+}\right) g \zeta+\sigma \partial^{2} \zeta / \partial x^{2}
$$

Here $\rho_{\text {. and }} \rho_{+}$are the densities of the lower (liquid) and the upper (gas) phase, respectively, $\varphi$ is the velocity potential, $g$ is the acceleration due to gravity, $\sigma$ is the interfacial tension and $\zeta$ the vertical elongation of the interface from its equilibrium position. In Eq. (1) we have assumed that $\zeta$ is varying only in the $x$ direction.

The dispersion relation for interfacial waves of frequency $\omega / 2 \pi$ and wave vector $q$, derived from Eq. (1), is

$$
\left(\rho_{-}+\rho_{+}\right) \omega^{2}=\left(\rho_{-}-\rho_{+}\right) g q+\sigma q^{3}
$$


where we have made the simplifying assumption, fulfilled in the following sections (except for the helium films in section 7) that the distances $d$. and $d_{+}$of the surface from the container bottom and top are large enough that $\tanh \left(d_{+} q\right) \cong \tanh \left(d_{-} q\right) \cong 1$.

For small $q$ the first term on the right-hand side of Eq. (2) dominates, yielding $\omega \propto q^{1 / 2}$ for these "gravity waves"; for large $q$ the dispersion of "capillary waves", $\omega \propto$ $q^{3 / 2}$, is obtained. The transition between these two types of waves occurs around $q=1 / a$ where

$$
a=\left[\sigma /\left(\rho_{-}-\rho_{+}\right) g\right]^{1 / 2}
$$

is the capillary length, describing the typical lateral range of static deformations of the interface. Incidentally, the phase velocity $\mathrm{v}_{\mathrm{ph}}$ of interfacial waves has a minimum at $q=1 / a$; for a charged interface the importance of waves with this particular wave vector will become more evident further below.

So far the damping of the ripplons due to the viscosity of the bulk liquid has been neglected. A more complete treatment shows that damping modifies Eq. (2), leading to a somewhat complicated implicit equation for the dispersion relation ${ }^{5,6}$. Since in the region of interest the resulting correction of the ripplon frequencies is less than $5 \%$, we omit here damping for the sake of simplicity.

Eqs. (1)-(3) not only hold for the "free" liquid surface (i.e., the liquid-gas interface) of helium, but also for the liquid-liquid interface of phase-separated ${ }^{3} \mathrm{He}-{ }^{4} \mathrm{He}$ mixtures below the tricritical point ${ }^{5}$, and, in a slightly modified way, also for the interface between superfluid and solid helium ${ }^{7,8}$. These systems will be used, in addition to the free liquid helium surface, in some of the examples below to illustrate the coupling of charges and interfacial modes.

Charged surface. When the surface is charged, the electrons under the influence of the (homogeneous) external electric field exert a pressure upon the interface. For a completely uniform charge distribution this pressure also is uniform. Any local deformation of the interface, however, results in a rearrangement of the electrons, which are accumulated in the troughs and depleted in the crests of a, for example, sinusoidal perturbation. Therefore, the local pressure in the troughs is higher and in the crests is lower than average. The electrons thus tend to increase any deformation of the interface. Consequently, in the equation of motion of such a charged interface an additional term appears due to the pressure arising from the discontinuity in the electric field perpendicular to the interface

$$
\left(\rho_{-}+\rho_{+}\right) \partial \varphi / \partial t=-\left(\rho_{-}-\rho_{+}\right) g \zeta+\sigma \partial^{2} \zeta / \partial x^{2}+\left\{\left[E_{+}(x)\right]^{2}-\left[E_{-}(x)\right]^{2}\right\} / 8 \pi
$$

where the (+) and (-) signs again refer to the upper and lower phase. We shall assume in the following that the interface is charged to the saturation density $n_{\mathbf{s}}$, i.e. the average field in the upper phase is screened completely, whereas in the lower phase it is

$$
E_{0}=4 \pi n_{s} \mathrm{e}
$$


(e is the elementary charge). Furthermore, effects due to the finite mobility of the electrons along the surface will be neglected here ${ }^{(1)}$. One then obtains for the dispersion relation of a charged interface ${ }^{10,11}$

$$
\left(\rho_{-}+\rho_{+}\right) \omega^{2}=\left(\rho_{-}-\rho_{+}\right) g q+\sigma q^{3}-4 \pi\left(n_{3} \mathrm{e}\right)^{2} q^{2}
$$

From Eq. (6) it follows that the aforementioned instability of the interface, related to the vanishing ripplon frequency $\omega\left(q_{c}\right) \rightarrow 0$, appears at a critical charge density $n_{c} \mathrm{e}=$ $\left[\left(\rho_{-}-\rho_{+}\right) g \sigma / 4 \pi^{2}\right]^{1 / 4}$, corresponding to a critical field

$$
E_{c}=\left[64 \pi^{2}\left(\rho_{-}-\rho_{+}\right) g \sigma\right]^{1 / 4}
$$

The wave vector where this instability develops is $q_{c}=\left[\left(\rho_{-}-\rho_{+}\right) g / \sigma\right]^{1 / 2}$, equal to the inverse capillary length defined in Eq. (3). Characteristic values for these quantities, calculated for the ${ }^{4} \mathrm{He}$ surface at $\mathrm{T}=2.5 \mathrm{~K}$, are $n_{c}=2.1 \times 10^{9} \mathrm{~cm}^{-2}, E_{c}=3700 \mathrm{~V} / \mathrm{cm}$, and $q_{c}=23 \mathrm{~cm}^{-1}$.

Experiment. The first measurement of the excitation spectrum of a charged He surface has been carried out with phase-separated ${ }^{3} \mathrm{He}-{ }^{4} \mathrm{He}$ mixtures ${ }^{12}$. There the interface between the superfluid ${ }^{4} \mathrm{He}$ - rich and the normal ${ }^{3} \mathrm{He}$ - rich phase allows one to trap both negative "ions" (= single electron bubbles) and positive ones ( "helium snowballs") ${ }^{13,14}$, and in addition the essential material parameters entering thetreatment sketched above

(the densities of the upper and the lower phase as well as the interfacial tension) can easily be varied by approaching the tricritical point ${ }^{15}$. In spite of the somewhat different system the results presented below should apply in a similar way also to coupled ripplon-plasmon modes at the free helium surface.

Fig. 1 shows a sketch of the sample cell. Negative and positive ions could be studied alternatively. They were generated by a field emission tip or a radioactive source, and then were drawn towards the interface by the externally applied field, where they formed the two-dimensional Coulomb system. Generation of ripplons on the charged interface was achieved by a horizontal $20-\mu \mathrm{m}$-thick wire mounted slightly below the interface. The electric $d c$ potential of this wire was set such that the electric holding field and therefore the ion distribution were not perturbed by the presence of the wire. When a small $a c$ voltage $U_{s}$ (frequency $\omega_{\mathrm{ad}} / 2 \pi$ ) was superimposed on the $d c$ voltage, the pressure of the ions acting on the interface was modulated close to the wire, thereby exciting linear interfacial waves of frequency $\omega_{\mathrm{ad}} / 2 \pi$. The shortest waves that could be generated in this way were of the order of the distance between the wire and the interface, about $0.1 \mathrm{~mm}$; the interesting region of the dispersion relation, namely wavelengths of the order of $2 \pi a \approx 1 \mathrm{~mm}$, was therefore easily accessible. For the detection of the surface waves an optical method was used, based on the deflection of a focussed laser beam reflected off the oscillating interface. Fig. 2 shows an image of the profile of a surface wave determined in this way.

\footnotetext{
${ }^{1}$ A more extensive treatment is given in Ref. 9.
} 


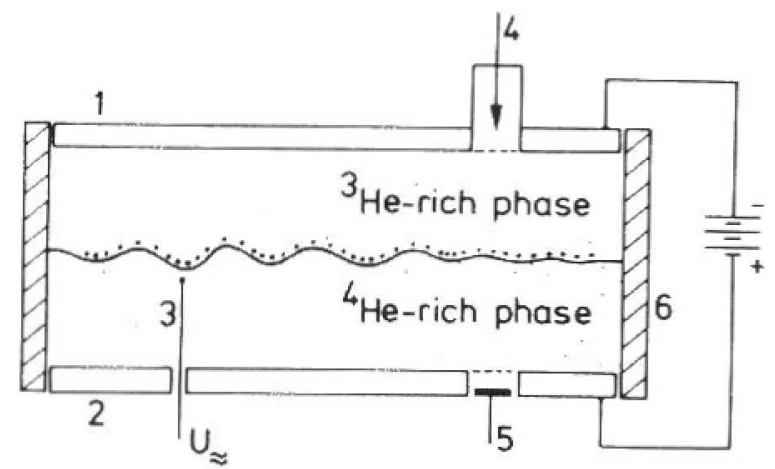

Figure 1: Sketch of the sample cell used for measuring the excitation spectrum of a charged ${ }^{3} \mathrm{He}-{ }^{4} \mathrm{He}$ mixture interface. (1) and (2) are the top and bottom capacitor plates. The wire of the wave generator (3) is perpendicular to the plane of the paper, as is the plane of incidence of the scanned laser beam. In the example drawn the interface is charged with negative ions from a field emission tip (4); for positive ions a radioactive source (5) is used. The guard ring (6) is charged such that the ions cannot escape to the walls of the cell. [Ref. 12]

Data for excitation spectra of a charged interface are plotted in Fig. 3. Compared to the neutral interface the ripplon frequencies with charges are distinctly reduced. This effect is more pronounced the closer the field is to the critical value $E_{\text {o }}$ $(875 \mathrm{~V} / \mathrm{cm}$ in this example). Apparently the largest influence on the dispersion relation occurs for wave vectors of the order of $1 / a=82 \mathrm{~cm}^{-1}$. The lines in Fig. 3 indicate the behavior expected according to Eq. (6) and are in good agreement with the data.

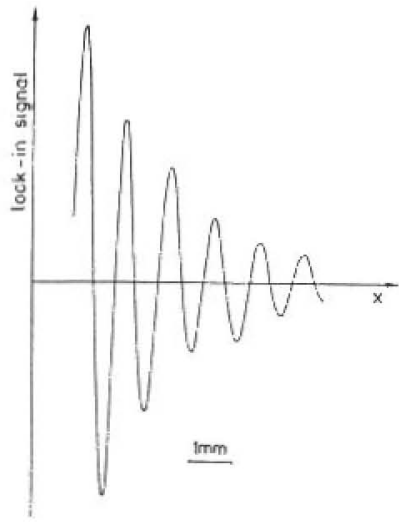

Figure 2: Interfacial wave at a charged ${ }^{3} \mathrm{He}-{ }^{4} \mathrm{He}$ interface, excited as shown in Fig. 1. The signal was obtained by focussing a laser beam onto the moving interface and detecting the oscillation of the reflected beam by a position-sensitive photo cell and a phase-sensitive amplifier. As the laser spot is scanned across the interface, the output signal represents a momentary picture of the interfacial wave and provides both the wavelength and the damping of that wave. The maximum wave amplitude is $5 \mu \mathrm{m}$. [After Ref. 12] 


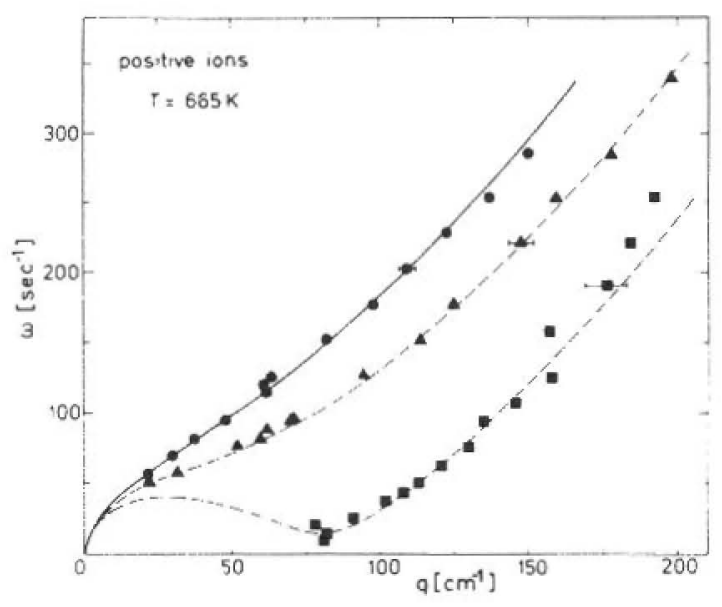

Figure 3: Dispersion relation of ripplons at the interface of a phase-separated ${ }^{3} \mathrm{He}-{ }^{4} \mathrm{He}$ mixture, completely charged with positive ions from below, at $T=0.665 \mathrm{~K}$ (taken from Ref. 12). The interface becomes unstable at $E_{\mathrm{c}}=875 \mathrm{~V} / \mathrm{cm}$, corresponding to an ion density $n_{c}=4.8 \times 10^{8} \mathrm{~cm}^{-2} . \bullet, E / E_{c}=0.12$; $40.71 ; 0.995$. The dashed curves are calculated according to $\mathrm{Eq}$. (6). The dispersion curve of the uncharged interface is given by the solid line.

The theoretical excitation spectrum (6) displays a remarkable feature: For $E=$ $(3 / 4)^{1 / 4} E_{c}$ it has an inflection point with a horizontal tangent, and for larger values of $E$ a region is found where three different $q$-vectors exist for each frequency. Since in the measurement the frequency was the quantity that was fixed externally, it was not clear offhand whether only one or possibly more $q$-vectors would be excited simultaneously. Experimentally, it was found that the wave pattern in that range was actually different from the usual exponential decay displayed in Fig. 2. The mode with the highest $q$ appeared most clearly, but an additional modulation was present which might be interpreted as the mode of the branch with negative group velocity. An unambiguous identification turned out to be difficult because damping of the waves was considerably increased in that region. For accurate measurements of waves below $q=20 \mathrm{~cm}^{-1}$, where the group velocity $\partial \omega / \partial q$ becomes positive again, the sample chamber was not big enough.

Of the ripplons investigated, those with the critical wave vector $q_{c}=1 / a$ are of particular interest, since they should finally lead to an instability of the interface at $E=E_{\sigma}$ As the electric field is increased, the ripplon frequency should be reduced, according to Eq. (6), to

$$
\omega\left(q=q_{c}\right)=\omega_{0}\left(q=q_{c}\right)\left[1-\left(E / E_{c}\right)^{2}\right]^{1 / 2}
$$

where $\omega_{0}$ is the ripplon frequency at $q=q_{c}$ for the uncharged interface. Experimental results for the electric field dependence of ripplons at the critical wave vector are given 
in Fig. 4. Again, the line shows the expected behavior (Eq. (8)). Due to the softening the power spectrum of spontaneous oscillations (noise) of a charged liquid helium surface should display a maximum corresponding to surface excitations with a wave number of the order of the soft ripplon mode. This was indeed observed experimentally ${ }^{16}$.

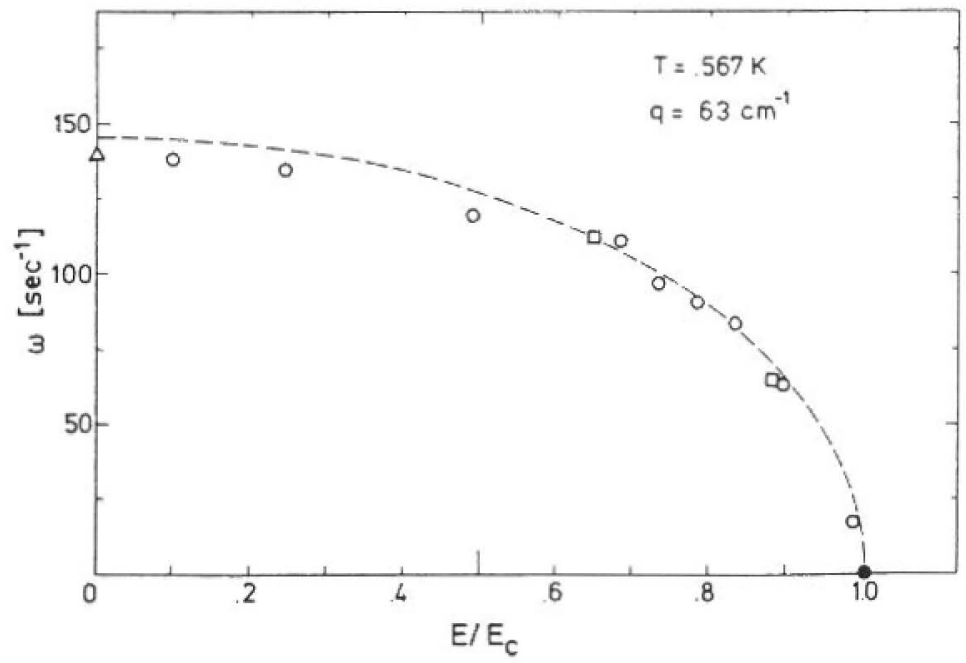

Figure 4: Frequency of interfacial waves in ${ }^{3} \mathrm{He}-{ }^{4} \mathrm{He}$ at a temperature $T=0.567 \mathrm{~K}$ and a wave vector $q_{c}=1 / a=63 \mathrm{~cm}^{-1}$. The interface is always charged to saturation. Circles represent measurements with positive and squares those with negative ions. The dashed line shows the result of Eq. (8). The discrepancy between this curve and the datum point at $E=0$ (uncharged interface) is due to the damping of the interfacial waves. [Ref. 12]

Fig. 4 bears strong resemblance to the softening of phonons, as it is observed, for example, for structural phase transitions. The continuous variation of $\omega$, with $\omega \rightarrow$ 0 for $E \rightarrow E_{c}$, is already a signature suggesting that this instability can be regarded as a type of second-order or weakly first-order phase transition (see below).

\section{The Dimple Lattice}

As shown, the frequency of the soft ripplon mode at $q=1 / a \equiv q_{c}$ vanishes when the electron density reaches a critical value $n_{c}$. For $n$ just above $n_{c}$ Eq. (6) predicts $\omega$ to become imaginary at a critical wave vector $q=q_{c}$, which implies that fluctations in the elongation of the interface with that particular $q$-value will increase in time instead of decay. As a result, a disruptive loss of charge from the surface develops, connected with a strong perturbation of the liquid ${ }^{17}$. 

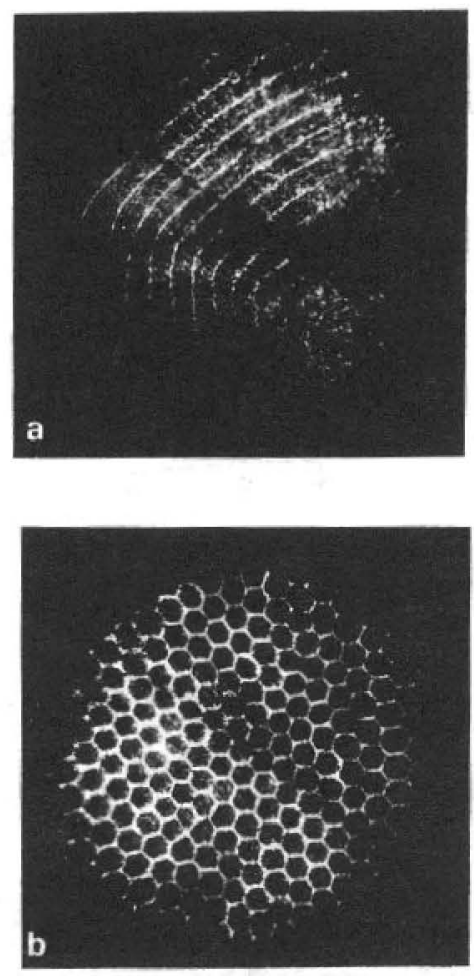

Figure 5: Formation of a dimple lattice on a free ${ }^{4} \mathrm{He}$ surface $(T=3.5 \mathrm{~K})$ charged with electrons from above. The pictures, which were taken through the transparent top electrode of the sample cell capacitor, show the surface deformation approximately $2 \mathrm{~s}$ (a) and $6 \mathrm{~s} \mathrm{(b)} \mathrm{and} \mathrm{(c))} \mathrm{after} \mathrm{the} \mathrm{field} \mathrm{had} \mathrm{been} \mathrm{increased}$ to $E_{\mathrm{c}}$. The image plane in (a) and (b) was chosen such that convex deformations of the surface, corresponding to local maxima, appear bright; in (c) bright areas correspondend to local minima (i.e. the center of the dimples). The distance between adjacent rows of dimples is close to the wavelength $2 \pi a$ of the soft ripplon, $0.24 \mathrm{~cm}$ in this case. [After Ref. 19]

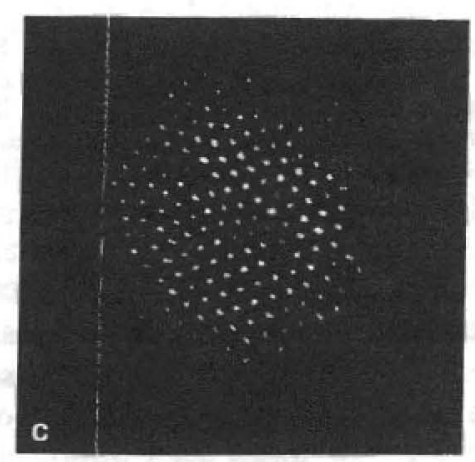

A qualitatively quite different feature of the $\mathrm{EHD}$ instability is observed, when the surface is not charged to saturation, but to only $8 \%$ of $n_{c}$ or less ${ }^{18}$. Although again interfacial waves with $q=q_{c}$ become unstable as the electric field is raised somewhat above a critical value $E_{c}$ (and consequently the interface ripples spontaneously with a characteristic wavelength, which is again $\lambda_{c}=2 \pi a$ ), nonlinear contributions to the restoring force stabilize these deformations at a finite amplitude. Thus a new equilibrium state in the charge distribution develops, different from the homogeneous layer of electrons for fields below $E_{c}$. 
The formation of the new state typically occurs in two steps, as illustrated in Fig. 5 for the case of a free ${ }^{4} \mathrm{He}$ surface (in these photographs, taken by a Schlieren technique, local maxima (Figs. 5a, 5b) or minima (5c) of the deformed surface appear bright). First, a standing wave-like pattern with $q=q_{c}$ develops, which then breaks up further into a periodic array of macroscopic dimples. Simultaneously the electrons, being pulled into local minima of the surface by the externally applied field, first accumulate in the wave troughs and then at the centres of the dimples. The typical duration of the whole process is a few seconds. Since the resulting triangular "dimple crystal" has developed from the soft ripplon mode, its lattice spacing is close to $\lambda_{\mathrm{c}}$.

Both $\lambda_{\mathrm{c}}$ and the critical field $E_{c}$ depend on the material parameters of the helium substrate and therefore on temperature ${ }^{18}$. Fig. 6 shows results for the critical field of a charged ${ }^{4} \mathrm{He}$-surface, which follow the temperature dependence of the parameter $\left[\left(\rho_{-}-\rho_{+}\right) \sigma\right]^{1 / 4}$ as expected from Eq. (7).

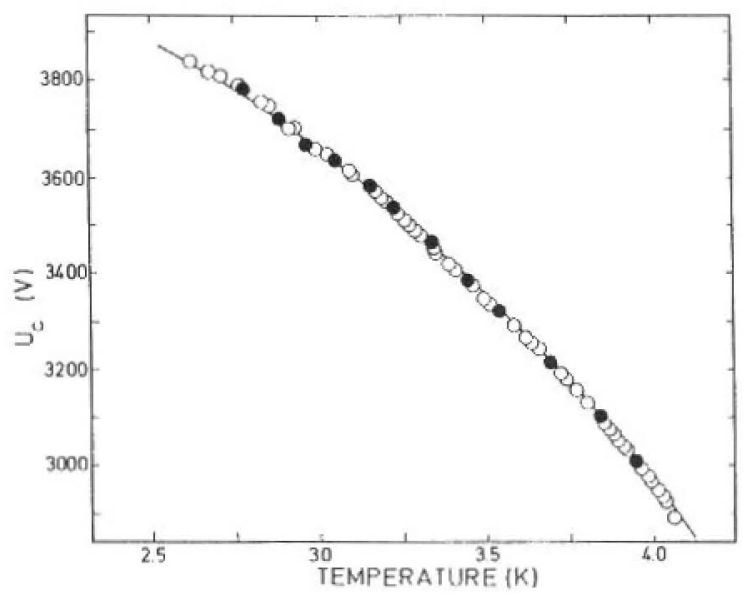

Figure 6: Critical voltage $U_{c}$ for the formation of the dimple lattice on liquid ${ }^{4} \mathrm{He}$ (the distance between the capacitor plates was $1.5 \mathrm{~cm}$ ). Open and closed symbols refer to different experimental runs. The hysteresis $\Delta U$ between the appearance and disappearance of the dimple lattice for increasing and decreasing voltage, respectively, is smaller than the symbol size. [Ref. 18]

The transition to the dimple state is reversible: By reducing the electric field the homogeneous charge distribution and the flat interface are retained. If the charge density is lower than the above-mentioned limit of $0.08 n_{\varepsilon}$, the system can repetitively be cycled through the instability without a loss of charge. An analysis of this transition shows that - in terms of the language of phase transitions - it is of weakly first $\operatorname{order}^{20,21}$. Consequently, hysteresis effects in the dimple formation are expected on varying the electric field $E$, which here plays the role of an effective temperature. Such effects have been observed indeed in a range $\mid\left(1-\left(E / E_{c}\right) \mid\right.$ of the order of $10^{-2} \cdot 18,21$ 
Furthermore, in an experiment where the liquid surface was carefully shielded from external vibrations, a striped phase has been found to be stable in a certain range close to $E_{\mathrm{c}}{ }^{22}$, which has also been accounted for by theory ${ }^{23}$.

Another interesting property of the dimple lattice follows from a consideration of the charge distribution in this state: As shown below in section 4 the charge density is not just slightly modulated along the surface, but is large in a region close to the dimple centres and zero elsewhere ${ }^{9,19,23,24}$. Since the potential barrier between neighboring dimples is high, the amount of charge of each dimple is fixed, and as a result the interaction between the dimples is dominated by Coulomb repulsion with only a small contribution from the deformation of the interface. The dimple lattice can therefore be regarded as an example of a two-dimensional Coulomb crystal, and is in many respects a macroscopic counterpart of the (microscopic) Wigner crystal introduced in previous chapters. Lattices like the one in Fig. 5 not only have the hexagonal symmetry expected for such 2D systems consisting of particles with isotropic interaction, but even display typical defects like dislocations and grain boundaries. ${ }^{19,22}$

In view of the similarilies between the microscopic and the macroscopic Coulomb systems one might also think of studying the melting of the dimple crystal (i.e., the transition to a disordered liquid-like phase at higher temperatures) to learn about the underlying mechanisms of such a phase transition. In analogy to the microscopic Wigner crystal one would expect this transition to be of the KosterlitzThouless type. As an attractive experimental advantage the dimple system would allow directly the observation of the unbinding of dislocation and disclination pairs, which are essential features for this kind of phase transition. One can easily convince oneself, however, that the melting temperature in this case would be higher than $10^{3} \mathrm{~K}$, due to the strong Coulomb interaction of the dimples. Hence thermodynamic melting phenomena are unfortunately not accessible with the dimple system ${ }^{(2)}$.

Another aspect are the collective excitations of a dimple lattice, which should be related to the longitudinal and transverse plasmons in a microscopic 2D electron system, yet with modifications caused by the coupling of the charges to the surface. In fact, preliminary observations demonstrate the existence of longitudinal modes of the dimple lattice, which are distinctly higher in frequency than the Rayleigh wave with the same wavelength at the uncharged surface ${ }^{25}$. More detailed investigations of the dynamics of the dimple system are still to be carried out.

\section{Individual Multielectron Dimples}

The existence of multielectron dimples on liquid helium does not depend on the formation of a more or less regular array. If only few electrons are present at the surface and the electric field is chosen properly, dimples can develop and are stable also as single, isolated objects. The stability and characteristic properties like width, depth, and eigenfrequencies of such individual dimples have been determined by a number of authors ${ }^{9,19,23-29}$. Typically, a dimple containing $5 \times 10^{6}$ electrons is a few

\footnotetext{
${ }^{2}$ It is possible, however, to use external perturbations, e.g. sufficiently strong $a c$ fields, to destroy the lattice and "melt" the dimple crystal (Ref. 25).
} 
tenths of a mm deep and has a width on the order of the capillary length, which is in the $\mathrm{mm}$ range. It should be mentioned that like the ripplon softening also dimple formation is not restricted to the free surface of liquid $\mathrm{He}$, but has been observed for the liquid-liquid and liquid-solid interfaces of condensed helium as well.

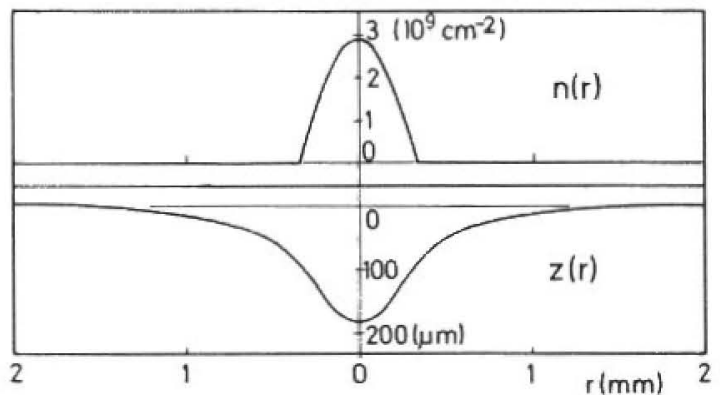

Figure 7: Calculated electron density $n(r)$ and surface profile $z(r)$ of a dimple containing $5 \times 10^{6}$ electrons in an electric field $E=3400 \mathrm{~V} / \mathrm{cm}$. The ${ }^{4} \mathrm{He}$ temperature is $2.5 \mathrm{~K}$, the corresponding critical field in this case, where in contrast to the condition of $\mathrm{Eq} .(7)$ the field in the upper half space is not shielded, is $E_{\mathrm{c}}=\left[16 \pi^{2}\left(\rho .-\rho_{+}\right) g \sigma\right]^{1 / 4}=2600 \mathrm{~V} / \mathrm{cm}$. [Ref.19]

An example for the surface profile and charge distribution of such an isolated dimple, investigated by means of a self-consistent numerical calculation ${ }^{19}$, is shown in Fig. 7. Starting from some fixed initial configuration of electrons, $n_{0}(r)$, and an external field $E$, the local depression was obtained by minimizing the total energy of the dimple, which consists of contributions from gravity, surface tension, electrostatic pressure and the Coulomb interaction of the electrons:

$$
W_{t o t} \approx \int\left\{1 / 2 \rho g z^{2}+\sigma\left[\left(1+z^{2}\right)^{1 / 2}-1\right]+z n_{0}(r) \mathrm{e} E+w_{\text {Coulomb }}\right\} 2 \pi r \mathrm{~d} r
$$

From the surface deformation found in this way a new electron configuration $n_{1}(r)$ was determined using the condition of constant electrostatic potential within the charge distribution and keeping the total number of electrons in the dimple $N=\int n(r) 2 \pi r \mathrm{~d} r$ fixed. In the next iteration step the whole procedure was repeated. The stability of such a localized charge distribution on the surface of liquid helium depends on the applied electric field and the number of electrons in the dimple:

i) At high fields and large $N$ the charges become more concentrated at the centre of the dimple in each iteration step, and the dimple depth increases indefinitely, leading to a break-through of the charges as expected.

ii) At low fields the charges spread out more and more, indicating the formation of a homogeneously distributed layer of electrons at the surface. 
iii) For an intermediate range of fields above $E_{c}$, a stable configuration is obtained after several iterations if the total number of electrons in the dimple is below a limit $N_{c}(E)$. (Note that in Fig. 7 the electron density $n(r)$ in the centre of the dimple is larger than the maximum attainable electron density $n_{c}=2.1 \times 10^{9} \mathrm{~cm}^{-2}$ of a homogeneously charged flat surface at the same temperature). The largest value of $N$ that was found to yield stable results for the liquid ${ }^{4} \mathrm{He}$ substrate was $10^{7}$ electrons per dimple. Taking into account that in the dimple lattice under the same conditions one has about 15 dimples $/ \mathrm{cm}^{2}$, this number compares well with the result of section 3 that the maximum electron density that could reversibly be cycled through the dimple instability is about $0.08 n_{c}$ or $1.7 \times 10^{8} \mathrm{~cm}^{-2}$.

Experimentally, the deformation of the liquid surface near a dimple was determined by analyzing the interference pattern of light reflected from the top and from the bottom capacitor plate. Fig. 8 shows an example of such a pattern and the corresponding dimple profile. Although the number of electrons in this dimple was not accurately known and hence a quantitative comparison with the numerical calculations was not possible, the shapes of calculated and experimental dimple profiles (Fig. 7 and Fig. 8) are obviously very similar.
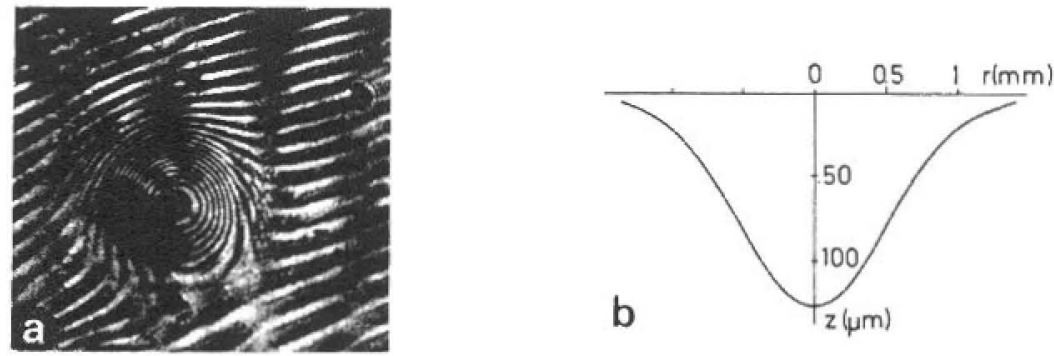

Figure 8: (a) Interference pattern of a macroscopic dimple on liquid ${ }^{4} \mathrm{He}\left(\mathrm{N} \sim 5 \times 10^{6}\right.$ electrons, $T=2.5$ $\mathrm{K}, E=2.9 \times 10^{3} \mathrm{~V} / \mathrm{cm}$ ). The fringe pattern is asymmetric because the two interferometer plates were slightly tilted. Consecutive fringes correspond to a difference in surface height of $10.9 \mu \mathrm{m}$. (b) The surface profile derived from (a). [Ref. 19]

Once a dimple is formed, its profile varies as the electrical field is raised further: The depth increases, and its width shrinks, so that the whole structure becomes sharper (see Fig. 9). Consequently at higher fields the electrons become more and more concentrated at the dimple centre. When the dimple profile is known, the charge density $n(r=0)$ at the centre can be derived from the condition of equilibrium between gravitational, surface, and electrostatic pressures:

$$
n(r=0) \mathrm{e} E \approx 2 \sigma / R+\left(\rho_{-}-\rho_{+}\right) g h
$$


Here $R$ is the radius of surface curvature at the dimple centre, and $h$ is the depth of the dimple. Experimental results for the dimple profile confirm the conclusion from the
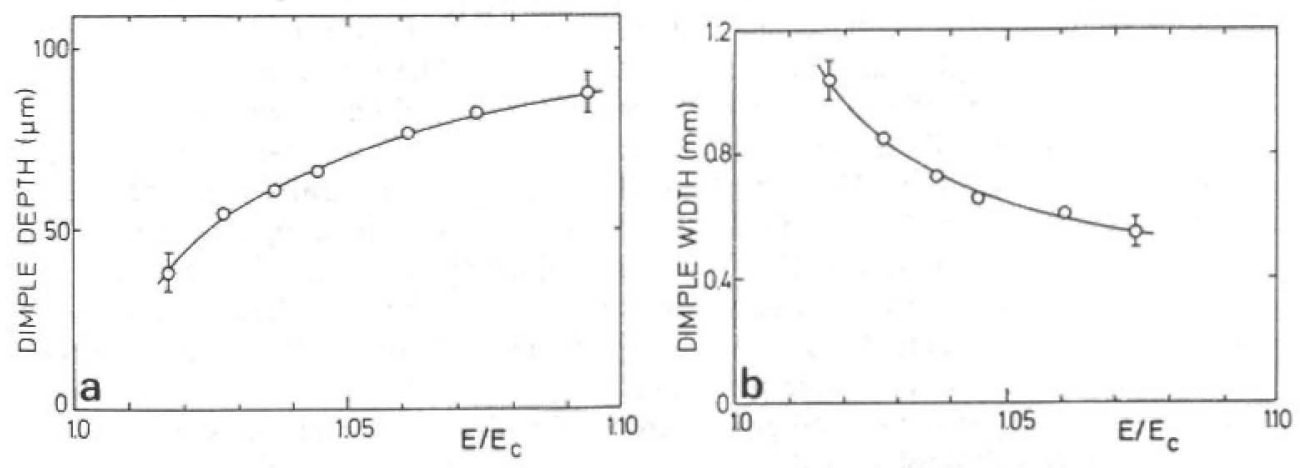

Figure 9: Experimental depth and width (full width at half minimum) of a dimple with constant charge vs. the reduced electric field $E / E_{\epsilon}(T=4.2 \mathrm{~K})$. The lines are only guides to the eye.[Ref. 19]

numerical calculation that at the dimple centre an electron density higher than $n_{c}$ (of the homogeneously charged surface) can be reached, which is stabilized by the surface tension of the curved He surface. It should be added that as an extension of the cylindrically symmetric dimple profiles considered so far also the more general case of elliptical dimples (caused by an anisotropy of the external potential or, in the case of ions at the solid-liquid interface, by the anisotropy of the crystalline substrate) has been studied ${ }^{27}$.
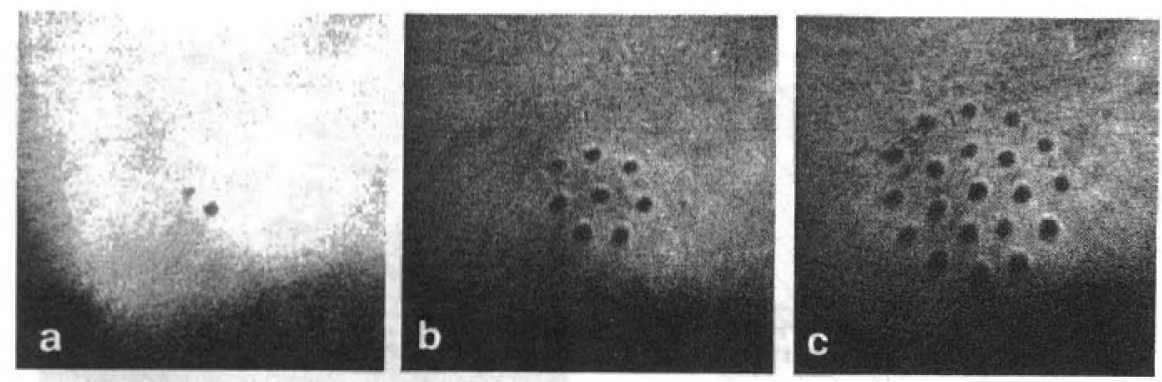

Figure 10: Configurations of (a) 2, (b) 8 and (c) 20 dimples (appearing as dark spots) in an external potential of cylindrical symmetry. In this experiment, the helium surface initially was uncharged and an electric field $E>E_{e}$ was applied. Then a pulse of electrons was generated by momentarily heating the electron source filament located close to the circumference of the sample cell. The emitted electrons were pulled against the surface and then formed a dimple with a charge $\mathrm{Ne}$ being determined by the emission of the source. Since a negative voltage, applied to the wall of the sample cell, provided a cylindrically symmetric potential well, the dimple after being generated drifted to the potential minimum at the centre of the cell. As more dimples were added they arranged themselves in circular shells, with a local structure approaching the hexagonal symmetry of the dimple lattice in Fig. 5. The size of each picture corresponds to $2 \mathrm{~cm}$. [Ref. 19] 
If two of the multielectron dimples are present at a surface, their interaction at large and small distances is governed by Coulomb repulsion, whereas at intermediate distances of a few capillary lengths the contribution caused by the surface energy could lead to a bound (stable or metastable) dimple pair ${ }^{9,23,30}$. Experimentally, pairs and larger clusters of dimples have been observed (Fig. 10). The presence of an external, laterally confining potential in this measurement makes it difficult, however, to draw conclusions about details of the dimple interaction. The fact that the average distance of the dimples in all the configurations of Fig. 10 is nearly constant could nevertheless be an indication for the existence of bound dimple states. The arrangement in shells, as seen in Fig. 10, is determined by the cylindrical symmetry of the confiningpotential, and bears close similarity to the arrays of classical Coulomb particles obtained in computer simulations by Peeters ${ }^{31}$. Upon further increasing the number of constituents the dimple configuration gradually develops into a hexagonal lattice. Apparently this is a second path to grow a dimple crystal, complementary to the formation via the soft ripplon mode as introduced in the previous section.

The dimples discussed so far were formed by electrons pushed against the helium surface from above. For charges trapped below the surface, the electrohydrodynamic instability ${ }^{32}$ and dimple formation can be observed as well, with surface profiles inverted as compared to Fig. 8. It is even possible to create dimple "dipoles", if at an applied electric field $E>E_{c}$ a surface is charged simultaneously from opposite sides with negative and positive charges. The schematic profile of such a dipole, consisting of a bound pair of oppositely charged dimples, is shown in Fig. 11. The binding energy of this complex is primarily provided by the Coulomb attraction of the two dimples. A recombination of electrons and positive ions does not take place, because, as for individual dimples, each kind of charge carriers is trapped close to the

a.

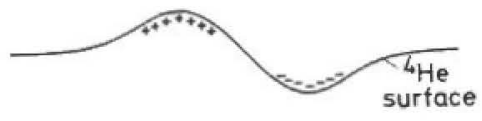

b.

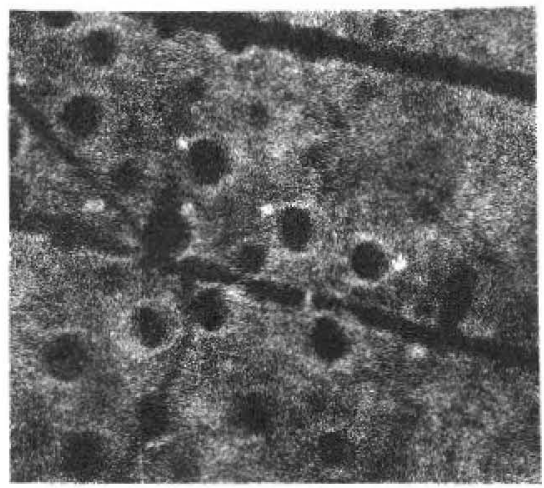

Figure 11: a) Schematic profile as observed for a bound pair of dimples with opposite charge.

b) Photograph of positively and negatively charged dimples at a "He surface. Due to the imaging the positive ion dimples appear as small bright spots, the electron dimples as larger dark disks. Most of the positive dimples (in the centre of the picture) are trapped by negative ones forming dipoles as sketched in a). [Refs. 19,25] 
centre of its corresponding dimple. However, the stability range of these dimple dipoles is distinctly less than for individual dimples (cf. Fig. 9), so that the positive and negative dimple quite easily "annihilate".

\section{The Electrohydrodynamic Instability at High Fields}

We now return to an initially homogeneously charged surface at a field $E<E_{c-}$. If in such a system the applied electric field is suddenly increased to a value well above $E_{c}$, the restoring forces that counteract any surface deformation are no longer sufficient to confine the charge at the interface. The resulting dynamics of the instability can be described, in its initial stage, by an extension of Eq. (6), which now in a certain band of wave vectors yields unstable solutions ${ }^{33}$. The range of unstable modes is plotted in the stability diagram in Fig. 12 as a function of the reduced electric field $E / E_{c}$ :

- for $E<E_{c}$ the system is stable for all values of $q$;

- at the instability threshold $E=E_{c}$ the mode with $q=q_{c}$ becomes unstable;

- for $E>E_{c}$ the unstable range, where a perturbation grows instead of decaying, covers an increasing band of wave vectors.

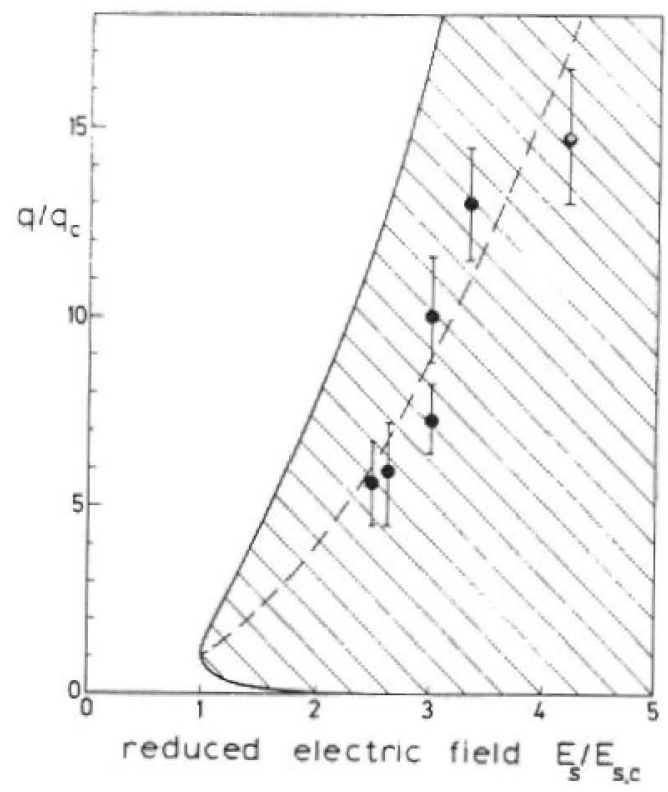

Figure 12: Stability diagram of a charged interface, as calculated from Eq. (6). The dashed line indicates the unstable wave vectors with the largest gain. The data points represent experimental results of $q_{\mathrm{m}}$ for the hep-superfluid ${ }^{4} \mathrm{He}$ interface. [Ref, 33] 
In general, the "punch through" process of the charges leads to a severe perturbation of the interface. The EHD deformations grow in amplitude, until the charged multielectron bubbles mentioned above split off and move to the counter electrode. For the liquid-gas interface this process, which can take place in a millisecond, has been recorded with a high speed camera ${ }^{17}$. Since the damping of surface waves in this case is small, the perturbations add up and the whole interface immediately is in chaotic motion (except if the geometry is chosen properly; see section 6 ). An interface which allows one to observe the development of the EHD instability under better-controlled conditions and in somewhat more detail is provided by the boundary between liquid and solid ${ }^{4} \mathrm{He}$. Excitations of this interface are propagating melting-crystallization waves ${ }^{7,8}$ whose dispersion relation is quite similar to that of ripplons at a liquid surface (Eq. (2)). The attenuation of these waves depends strongly on temperature; above $1 \mathrm{~K}$ they are overdamped in the critical wave vector range around the inverse capillary length ${ }^{34}$. When such an interface is charged (in this case not with quasi-free electrons, but with one-electron bubbles), the stability diagram shown in Fig. 12 should apply as before. As a technical advantage, however, in this case the perturbing influence of waves reflected from the container walls, which usually obscure the growth of the EHD instability, is suppressed because of the strong damping, and the growth of the unstable modes can easily be followed on a time scale of seconds.

A picture of such an interface between liquid and solid ${ }^{4} \mathrm{He}$, "quenched" into the unstable regime by a sudden increase of the electric field, is presented in Fig. 13.

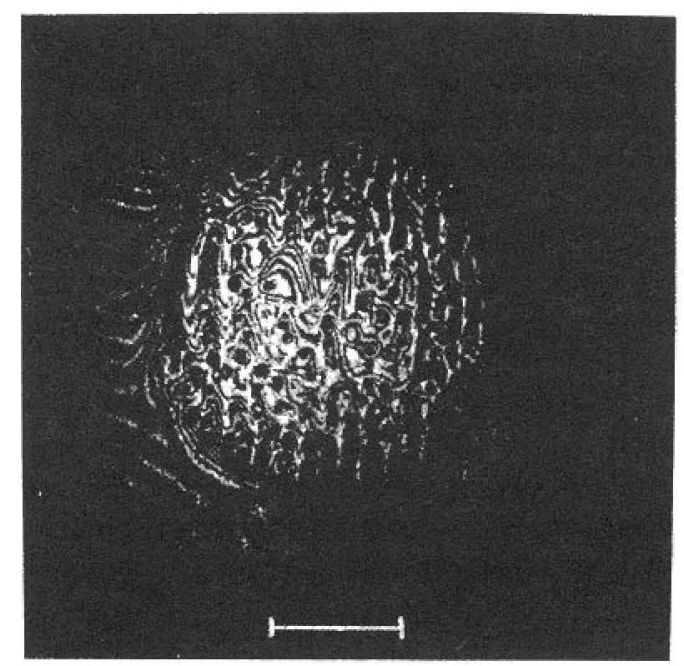

Figure 13: Snapshot of the instability pattern developing at an hcp-superfluid ${ }^{4} \mathrm{He}$ interface at $E / E_{c}=$ 2.6. The characteristic spacing of the corrugations, indicating the mode with the fastest growth, is distinctly smaller than the lattice spacing of the dimple crystal at the critical field, $2 \pi a=6.3 \mathrm{~mm}$ (indicated by the length of the scale bar). The size of the electron patch, determined by the confining field of the circular cell walls, was $20 \mathrm{~mm}$. [Ref. 33] 
Although the pattern is not nearly as regular as the dimple lattice in Fig. 5, some periodicity in the deformation of the crystal can still be recognized. Apparently a small range within the wide band of competing unstable modes at that field dominates the growth. The orientation of the corrugations visible in Fig. 13 is related to the anisotropy of the ${ }^{4} \mathrm{He}$ crystal.

The higher the applied field the faster this instability is found to develop. Simultaneously the wave vector $q_{m}$ characterizing the spacing of the corrugations becomes larger and less well-defined. Thus there is considerable scatter in the data points plotted in the stability diagram (Fig. 12). Nevertheless the agreement with the predicted curve for the modes with the fastest growth, shown as the dashed line in Fig. 12 , appears reasonable, indicating that the essential features of the phenomenon are covered by the theory sketched above.

The development of the EHD instability resembles closely, in certain respects, the phenomenon of spinodal decomposition in two-component liquids or alloys with a miscibility gap. When such a system is quenched from the one-phase state through the critical point into the unstable range, it starts to phase-separate spontaneously. Like for the EHD instability this process is characterized by the instability of a whole range of wave vectors, and a particular $q$-value grows most quickly and dominates the decomposition, resulting in patterns similar to Fig. 13. Thus again the charge-induced instability at helium interfaces can be considered in the context of a much wider class of critical phenomena.

\section{Multielectron Bubbles}

It has already been pointed out that the final stage of the EHD instability involves charged multielectron bubbles which break away from the interface and move towards the positive counter electrode. These bubbles (filled with gas or liquid, depending on the system to be studied) are stabilized by the interfacial tension $\sigma$ and the hydrostatic pressure $p$ in the surrounding phase, counterbalanced by the repulsive Coulomb forces between the electrons at the inside of the bubble. The equilibrium bubble radius is determined by minimizing the energy ${ }^{35,36}$

$$
W_{\text {bubble }}=4 \pi R^{2} \sigma+(4 / 3) \pi R^{3} p+\left(N^{2} \mathrm{e}^{2}\right) /(2 \varepsilon R)
$$

(accounting for surface energy, external pressure and electrostatic energy, respectively; $\varepsilon$ is the dielectric constant and $N$ e the total charge of the bubble). For typical values of $N$ between $10^{5}$ and $10^{7}$, Eq. (11) yields a diameter from 10 to $100 \mu \mathrm{m}$ for gas bubbles in liquid ${ }^{4} \mathrm{He}$ at svp.

These bubbles appear as interesting objects, because due to their mutual Coulomb repulsion the charges are confined to a thin "skin" close to the bubble surface and thus constitute, on a local scale, a 2D Coulomb system like the electrons on the flat liquid surface, however with a considerably higher density $\left(10^{10} \mathrm{e} / \mathrm{cm}^{2}\right.$ and more), again stabilized by the curvature of the He surface.

It has been demonstrated that it is possible to generate the bubbles in a controlled manner, and to study them by means of light scattering (see Fig. 14a) ${ }^{37}$. Fig. 
$14 \mathrm{~b}$ shows the current pulses due to a sequence of bubbles, $10^{6}$ electrons each, which was registered by means of an electrometer.
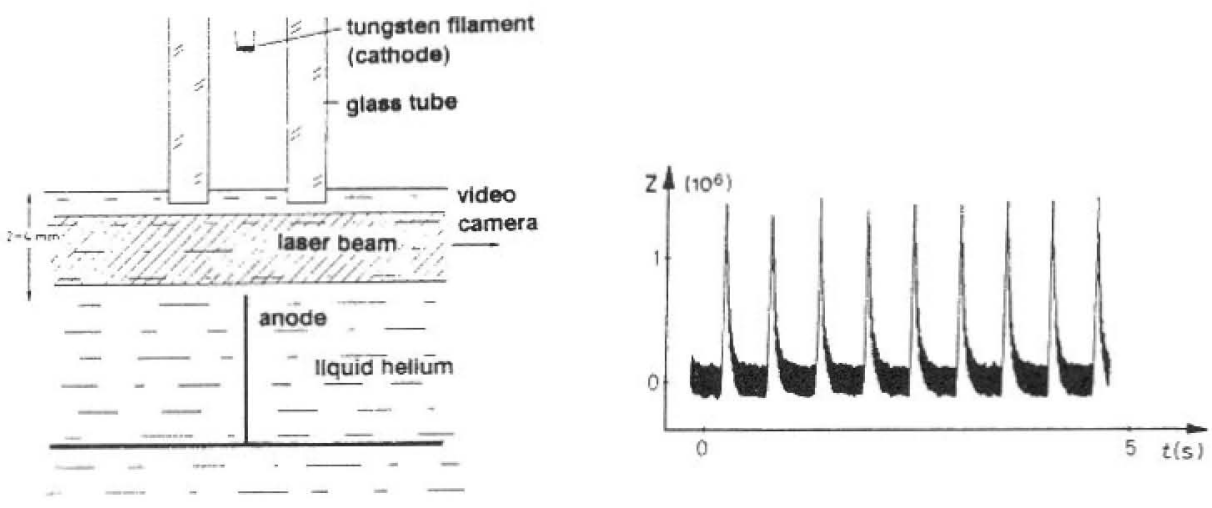

Figure 14: a) Schematic set-up for electrical and optical detection of multielectron bubbles. The video camera for the optical measurements was focussed on the volume between anode tip and glass tube, at an angle of $30^{\circ}$ with respect to the laser beam, to pick up light scattered in nearly forward direction. b) Displacement current at the anode recorded at a vapor pressure of 1000 mbar. Each peak corresponds to one multielectron bubble; the peak height is proportional to the charge of the bubble ( $Z$ : number of electrons in a bubble). [Ref. 37]

Using proper illumination these bubbles appear as spots with sufficient intensity to record their trace with a video camera. This is shown in Fig. 15, where the illuminating laser beam was chopped in order to determine the bubble velocity, $v$. It was found that $v$ reaches a constant value, typically $10 \mathrm{~cm} / \mathrm{s}$ under the conditions of Fig. 15, nearly instantaneously after the bubble has formed. This implies that the energy which the moving charges pick up in the applied electric field is quickly dissipated into other channels. One of them appears to be the excitation of bubble oscillations, which is supported by the observation that the brightness along the bubble paths fluctuates strongly even if instead of chopping the laser beam its intensity is kept constant. The observed oscillation frequencies are of the order of $2 \mathrm{kHz}$, which is in good agreement with calculated values for the lowest bubble mode ${ }^{35}$.

A promising aspect for further investigations comes from an experiment which has demonstrated that capture and storage of a bubble in an electric quadrupole configuration similar to an if trap for ions should be feasible ${ }^{37}$. This would enable one to study the eigenmodes of the bubbles in much more detail. Of particular interest in this context is the influence of the bubble surface charge on this mode, which is expected to reflect the changes of the electronic transport properties in the various regions of the phase diagram of the SSE system, particularly at high electron densities. 


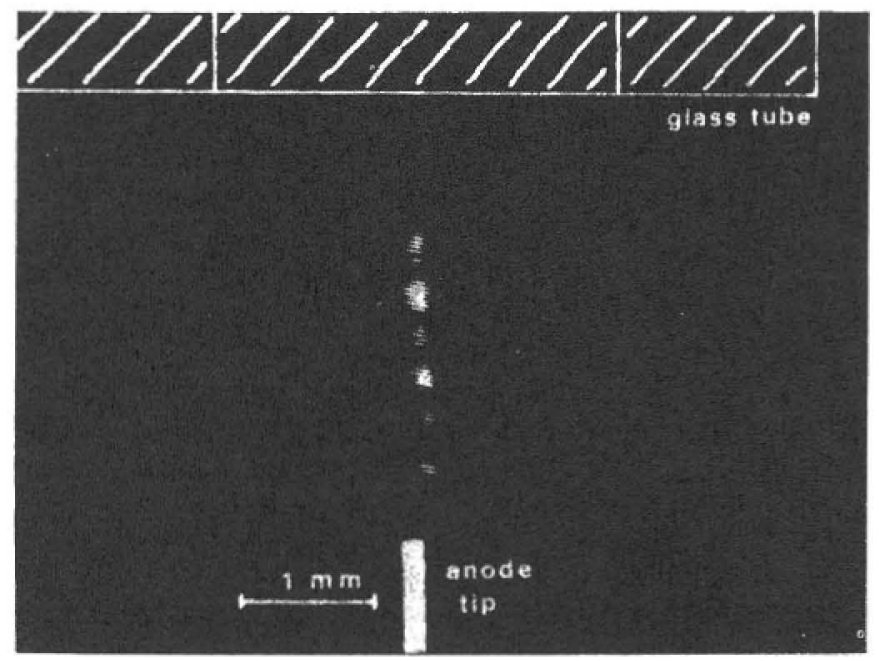

Figure 15: Track of a single multielectron bubble moving towards the anode tip. The irradiating laser is chopped at a frequency of $370 \mathrm{~Hz}$ to measure the velocity of the bubble. The size of the spots does not represent the size of the bubble, but only a measure for the intensity of the scattered light. [Ref. 37]

\section{Electrons on Helium Films}

An alternative route for reaching electron densities in excess of the limit imposed by the EHD instability of the flat bulk surface is to use a helium film as a substrate (see also chapter 13 of this volume). The stability of a film is increased due to van der Waals forces, as seen from the dispersion relation for ripplons coupled to the $2 \mathrm{D}$ electron layer $^{38-40(3)}$ :

$$
\rho \omega^{2}=\left(\rho_{s} / \rho\right)\left[\left(3 \alpha / d^{4}+\rho g\right) q+\sigma q^{3}-4 \pi\left(n_{s} e\right)^{2} q^{2} F(q \varepsilon)\right] \tanh (q d)
$$

Compared to the dispersion relation for a charged bulk helium surface, Eq. (6), there is an additional term which includes the van der Waals constant $\alpha$. The last factor on the $r h s$ of Eq.(12) arises from the finite depth $d$ of the liquid film, the function $F(q, \varepsilon)$ takes account of the image charges in the solid substrate, and the factor $\rho_{\mathrm{s}} / \rho$ indicates that in a thin He film only the superfluid component can move.

According to Eq.(12) charging a film reduces the ripplon frequency in a similar way as for bulk helium, but the critical charge density where the instability occurs is higher. For a thickness of $300 \AA$ - a typical value for a saturated film at a height $H=$ lcm above the bath level - it is expected that an electron density $n_{c}=3 \times 10^{10} \mathrm{~cm}^{-2}$ should be stable, one order of magnitude more than on bulk ${ }^{4} \mathrm{He}$.

${ }^{3}$ The contribution of the density of the gas phase, $\rho_{+}$, is neglected here. 
It was shown experimentally that not only is it possible to reach this limit, but that it can even be exceeded considerably ${ }^{41}$. It was found, for example, that saturated helium films on a hostaphan polymer foil could be charged with electrons up to a density of $0.98 \times 10^{11} \mathrm{~cm}^{-2}$ before break-through to the solid substrate set in. Moreover, this instability threshold appeared to be independent of the initial thickness $d_{0}$ of the uncharged films in the investigated range $200 \AA<d_{0}<400 \AA$.

This apparent discrepancy was resolved when the electronic pressure $p_{\mathrm{el}}$ exerted on the film was taken into account. Since $p_{\text {el }}$ adds to the gravitational and van der Waals terms in the chemical potential, the average equilibrium thickness of a charged film will be reduced to ${ }^{41}$

$$
d \cong d_{0}\left(1+2 \pi n^{2} \mathrm{e}^{2} / \rho g H\right)^{-1 / 3}
$$

assuming that the thickness before charging is given by $d_{\mathrm{o}}=(\alpha / \rho g H)^{1 / 3}$. As a result of the reduction of $d$ the instability threshold, calculated from Eq. (12), is raised, and a higher charge density can be obtained. A more detailed investigation has shown that if the separation of the electrons from the film due to quantum motion is taken into account, the charge saturated film is stable against surface perturbations for all densities $^{42}$.

The change in film thickness on charging was confirmed experimentally by an ellipsometric determination of $d^{41}$. Some data are plotted in Fig. 16 for two values of the initial thickness $d_{0}$. As expected from Eq. (13) the two curves converge for high electron densities. Consequently the stability limit for high electron densities on helium films is not significantly influenced by the initial film thickness.

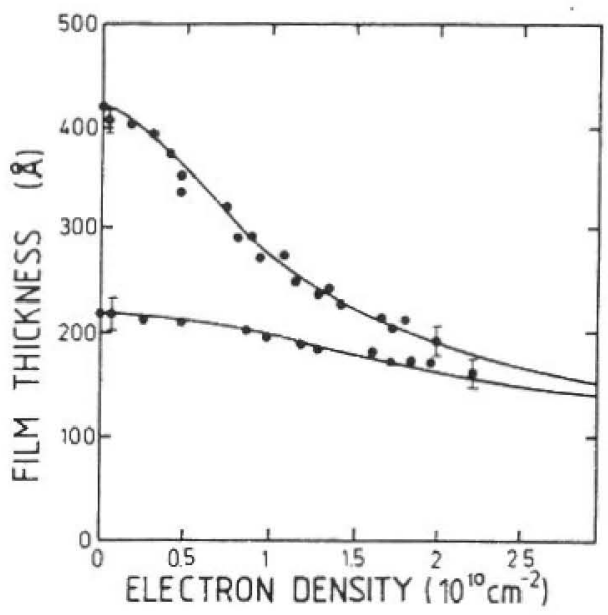

Figure 16: Thickness $d$ of charged saturated ${ }^{4} \mathrm{He}$ films wetting a glass substrate $(T=1.6 \mathrm{~K})$ versus the electron density on the films. The thickness $d_{0}$ of the uncharged films was 220 and $420 \AA$, respectively. [Ref. 41] 
What limits then the charge density on helium films? Eventually, when the film has become very thin ( $d \leq 50 \AA)$, tunneling of the electrons through the helium into surface states of the substrate will set in. This process might have been of importance for the highest densities reported so far, in particular since substrate inhomogeneities like surface roughness will lead to a locally reduced film thickness where tunneling can preferentially occur.

The high electron densities which can exist on helium films owing to the suppression of the EHD instability appear very promising for studies in the range where quantum effects modify or even dominate the behavior of the SSE system. For instance, recent microwave investigations have revealed anomalies in the conductivity and susceptibility of electrons on a helium film which have been considered as a signature of the predicted quantum melting of the Wigner $\mathrm{crystal}^{43}$. This phase transition and the properties of the 2D degenerate Fermi gas of SSE produced in this way are of considerable current interest. A new, broad field for future experiments with SSE on helium could thus become accessible.

\section{Conclusions}

The results presented here may give the impression that the electrohydrodynamic instability and the formation of multi-electron dimples at helium interfaces are quite thoroughly understood. For many features this is indeed the case: The SSE/helium system is probably the best-studied example for the coupling between electromagnetic and hydrodynamic modes at surfaces regarding not only the phenomena as such, but also the quantitative description. Yet there still exists a number of open questions. Little is known, for example, about charged films in the thickness range from nearly bulk material to van der Waals-stabilized films. Several theoretical papers on this subject have yet to be complemented by systematic experiments ${ }^{44}$. Likewise, the dynamics of the dimple lattice is hardly studied, and also the multielectron bubbles demand more detailed investigations. Recently, rather intriguing suggestions have been made to use surface deformations induced by SSE for decoration purposes, such as enhancing the surface profiles of vortices in rotating superfluid ${ }^{3} \mathrm{He}^{45}$, or imaging the potential distribution in quantum Hall devices ${ }^{46}$. Preliminary experiments show that SSE might be quite suitable for such decoration studies ${ }^{47}$. In any case, the coupled system of SSE and helium surface modes is as an instructive and transparent model for studying instability phenomena as well as an interesting and unique topic of ist own.

\section{Acknowledgements}

It is a pleasure to acknowledge numerous stimulating discussions with $\mathrm{E}$. Andrei, M.W. Cole, A. Dahm, P. Hakonen, V.B. Shikin and F.I.B. Williams. A. Dahm and V.B. Shikin have also been directly involved in some of the projects of our group, together with my collaborators and students W. Ebner, H. Etz, T. Günzler,W. Idstein, D. Savignac, E. Teske, and M. Wanner. Part of this work has been supported by the Deutsche Forschungsgemeinschaft. 


\section{REFERENCES}

1. See, e.g., Cole, M.W., RevMod.Phys. 46 (1974) p.451; Grimes, C.C., Surf.Sci. 73 (1978) p. 379; Edelman, V.S., Sov.Phys.Usp. 23 (1980) p. 227; Williams, F.I.B., Surf.Sci. 113 (1982) p. 371; Monarkha, Yu.P., and Shikin, V.B., Sov.J.Low Temp. Phys. 8 (1982) p. 279; Leiderer, P., Physica 126 B+C (1984) p. 92; Studart, N., and Hipolito, O., 1986, Revesta Brasileira de Fisica 16, p. 194; Shikin, V.B., and Monarkha, Yu.P., Two-dimensional charged systems in liquid helium, (Editorial board for physical and mathematical literature Moscow, 1989 ); Leiderer, P., J. Low Temp. Phys. 87 (1992), p. 247

2. Williams, R., and Crandall, R.S., Phys. Lett. 36A (1971) p. 35

3. Kovdrya, Yu.Z., and Monarkha, Yu.P.,Fiz. Nizk. Temp. 12 (1986), p. 1011

4. Landau, L.D. and Lifshitz, M., Fluid Mechanics, (Pergamon, Oxford, 1959), p. 237

5. Leiderer, P., Poisel, H. and Wanner, M., J. Low Temp. Phys, 28 (1976) p. 167

6. Bouchiat, M.A. and Meunier, J., J.Phys. (Paris) 32 (1971) p. 561; 33 (1972) C1-141

7. Andreev, A.F., and Parshin, A.Ya., Sov. Phys. JETP 48 (1978)p. 763

8. Keshishev, K.O., Parshin, A.Ya., Babkin, A.V., 1979, Sov. Phys. JETP 30 (1979) p. 56; Sov. Phys. JETP 53 (1981) p. 362

9. Shikin, V.B., and Leiderer, P., Sov. Phys. JETP 54 (1981) p. 92

10. Gorkov, L.P., and Chernikova, D.M., JETP Lett. 18 (1973) p. 68; Sov, Phys. Dokl. 21 (1976) p. 328

11. Mima, K., and Ikezi, H., Phys. Rev. B 17 (1978) p. 3567;

Mima, K., Ikezi, H., and Hasegawa, A., Phys. Rev. B14 (1976) p. 3953

12. Wanner, M., and Leiderer, P., Phys, Rev, Lett. 42 (1979) p. 315; Leiderer, P., Phys. Rev, B 20 (1979) p. 4511

13. Leiderer, P., Wanner, M., and Schoepe, W., J. Phys. (Paris), Colloq. 39 (1979) p. C 6-1328

14 For a review, see Fetter, A.L., in The Physics of Liquid and Solid Helium, eds, Bennemann, K.H., and Ketterson, J.B. (Wiley, New York, 1976), VoL. 1, p. 207

15. Leiderer, P., in Quantum Fluids and Solids, eds. Trickey, S.B., Adams, E.D., and Dufty, J.W., Plenum, New York, 1976) p. 351

16. Rybalko, A.S. Borzenets, V.V., and Grigorev, V.N., Fiz. Nizk. Temp. 9 (1983) p. 570

17. Volodin, A.P., Khaikin, M.S., and Edelman, V.S., Pis'ma Zh. Eksp. Teor. Fiz. 26 (1977) p. 707; (JETP Lett. 26 (1977) p. 543 );Khaikin, M.S., J.Phys. (Paris) 39 (1978) p. C6-1295

18. Leiderer, P., and Wanner, M., Phys. Lett. 73A (1979) p. 189; Ebner, W., and Leiderer, P., Phys. Lett. $80 \mathrm{~A}(1980)$ p. 277

19. Leiderer, P., Ebner, W., and Shikin, V.B., Surf. Sci. 113 (1982) p. 405; Physica 107B (1981) p. 217

Ikezi, H., Phys. Rev. Lett. 42 (1979) p. 1688

Chernikova, D.M., Fiz. Nizk. Temp. 6 (1980) p. 1513

Gianetta, R.W., and Ikezi, H., Phys. Rev. Lett. 47 (1981)p. 849; Surf. Sci. 113 (1982) p. 412

Ikezi, H., Gianetta, R.W., and Platzman, P.M., Phys. Rev. B25 (1982) p. 4488

Melnikov, V.I., Meshkov, S.V., Sov. Phys. JETP 54 (1981) p. 505

Ebner, W.; diploma thesis (Munich, 1980); Leiderer, P., unpublished

Nazin, S.S., and Shikin, V.B., Sov. Phys. JETP 58 (1983) p. 310

Shikin, V.B., and Leiderer, P., Sol. St. Commun. 47 (1983) p. 269; Shikin, V.B., Sov. Phys. JETP 59 (1984) p. 304

Degani, M.H., and Hipolito, O., J. Phys. C16 (1983) p. L1109

Shikina, N.I., Sol. State Commun. 51 (1984) p. 901

Krasni, Yu.P., Mikho, V.V., Fiz. Nizk. Temp. 11 (1985) p. 675

Bedanov, V.M., and Peeters, F., Phys. Rev. B49 (1994) p. 2667

Boyle, F.P., and Dahm, A.J., J. Low Temp. Phys. 23 (1976) p. 477

Savignac, D., Leiderer, P., Phys. Rev. Lett. 49 (1982) p. 1869

Bodensohn, J., Nicolai, K, and Leiderer, P., Z.Phys.B 64 (1986) p. 55

Williams, G.A., and Salomaa, M.M., J. Low. Temp. Phys. 57 (1984) p. 539; Salomaa, M.M., and Williams, G.A., Phys. Rev. Lett. 47 (1981) p. 1730

Shikin, V.B., JETP Lett. 27 (1978) p. 39

Albrecht, U., and Leiderer, P., Europhys. Lett. 3 (1987) p. 705

Ikezi, H., and Platzman, P.M., Phys. Rev. B 23 (1981) p. 1145

Peeters, F.M., and Platzman, P.M., Phys. Rev.Lett. 50 (1983) p. 2021 
40. Williams, G., private communication

41. Etz, H., Gombert, W., Idstein, W., and Leiderer, P., Phys. Rev. Lett. 53 (1984) p. 2567

42. Hu, X.L., and Dahm, A.J., Phys. Rev. B 42 (1990)p. 2010

43. Gunzler, T., Bitnar, B., Mistura, G., Neser, S., and Leiderer, P., Surf. Sci. to be published 44. Shikin, V.B., and Lebedeva, E., Z. Phys. B98 (1995) p. 331; Tatarskii, V.V., Shikina,

N.L., Shikin, V.B., Fiz. Nizk. Temp. 10 (1984) p. 117

45. Hakonen, P.J., Penttila, J.S., Sonin, E.B., to be published

46. Shikin, V.B., and Leiderer, P., to be published

47. Teske, E., diploma thesis (Konstanz, 1995) unpublished 